\title{
Nota
}

\section{Modelamiento de la distribución potencial de Corapipo leucorrhoa (Pipridae) en el valle alto del Magdalena, Tolima, Colombia}

\author{
Modeling the potential distribution of Corapipo leucorrhoa \\ (Pipridae) in the upper Magdalena Valley, Tolima, Colombia
}

\author{
Cristhian David Gaitán García ${ }^{\circledR}$, Sergio Losada Prado
}

\section{Resumen}

En los últimos años, el saltarín gorgiblanco (Corapipo leucorrhoa) ha sido reportado por fuera de su distribución habitual hacia el norte del valle alto del Magdalena (Tolima, Colombia), lo cual supone que su distribución real no es bien conocida. En este estudio se reportan tres registros de C. leucorrhoa en el departamento del Tolima. Con la revisión de varios registros de bases de datos y nuestros reportes, modelamos un mapa de distribución potencial para esta especie, encontrando que hay condiciones propicias para su presencia a lo largo del valle alto del Magdalena y que esta región debe ser incluida como parte de su distribución geográfica para Colombia.

Palabras clave. Ampliación de rango. Bosque húmedo premontano. Distribución geográfica. Pipridae. Río Amoyá.

\begin{abstract}
In recent years, the white-bibbed manakin (Corapipo leucorrhoa) has been reported outside its usual distribution north of the upper Magdalena valley (Tolima, Colombia), which means that its actual distribution is not well known. In this study, three records of C. leucorrhoa in the department of Tolima are reported. With the review of several database records and our reports, we modeled a potential distribution map for this species, finding that there are favorable conditions for its presence along the upper Magdalena valley, and that this region should be included as part of its geographical distribution for Colombia.
\end{abstract}

Keywords. Amoyá River. Geographical distribution. Pipridae. Premontane wet forest. Range extension. 


\section{Introducción}

El saltarín gorgiblanco (Corapipo leucorrhoa) es una especie poco común y local, con preferencia por el bosque húmedo primario, bosque ripario y vegetación en crecimiento secundario (Hilty \& Brown, 2001). Originalmente presentaba un rango geográfico amplio desde Honduras a Venezuela, con tres subespecies: C. leucorrhoa altera (norte de Costa Rica a Honduras), C. leucorrhoa heteroleuca (sur de Costa Rica, Panamá) y C. leucorrhoa leucorrhoa (Colombia y Venezuela; Rosselli et al., 2002). Sin embargo, varios autores las clasificaron como especies separadas, formando dos grupos: el primero elevando a especie a C. leucorrhoa altera, que incluye a C. leucorrhoa heteroleuca como subespecie de ella, y segundo, C. leucorrhoa leucorrhoa quedó como especie monotípica (Sibley \& Monroe, 1990; Ridgely \& Tudor, 1994; AOU, 1998; Remsen et al., 2019). C. leucorrhoa se distribuye entre los 200-1700 m s. n. m, y en Colombia se encuentra en la base norte de los Andes por el valle del río Magdalena hasta el norte del Tolima y también en el Valle del Cauca hasta Cali; al este de los Andes se encuentra en Norte de Santander, hasta la Serranía de Perijá y noroccidente de Arauca; en Venezuela se distribuye por las vertientes andinas bajas de Mérida, Barinas, Táchira y la Serranía del Perijá en la frontera Venezuela-Colombia (Restall et al., 2006; Ayerbe-Quiñones, 2018).

Las novedades taxonómicas mencionadas han hecho que los mapas de distribución se deban ajustar a la información más reciente; un ejemplo de esto son ChaparroHerrera et al. (2013) que catalogan a C. leucorrhoa como una especie casi endémica, con un rango geográfico más amplio que no se ajusta a los mapas de distribución más recientes (Ayerbe-Quiñones, 2018). Por ejemplo, ellos la incluyen al noroeste del Pacífico colombiano y Panamá, pero en realidad su distribución no incluye esas regiones, pues su rango geográfico es más restringido: más del $50 \%$ la distribución de esta especie está en Colombia. Otro escenario interesante es que en los últimos años ha habido varios reportes de C. leucorrhoa al sur de la distribución actual conocida. De acuerdo con diversos reportes en eBird (https:/ / ebird.org/ species/whbman2), hacia el valle alto del Magdalena hay registros en Paquiló (Cundinamarca) y para el departamento del Tolima hay varios registros en los municipios de Líbano y Fresno. Por lo tanto, estos registros hacen suponer que la distribución geográfica de esta especie podría ser más amplia de lo que se ha considerado, siendo necesario un modelamiento para actualizarla.
Por eso en este estudio, a través del reporte en tres localidades recientes en el departamento del Tolima y los registros históricos para C. leucorrhoa, proponemos un nuevo mapa de distribución potencial para esta especie en Colombia.

\section{Materiales y métodos}

Los reportes de C. leucorrhoa fueron obtenidos con diferentes fechas y métodos de muestreo en tres localidades diferentes (Figura 1), con zonas de vida de bosque seco tropical y bosque húmedo premontano según la clasificación de Holdridge (1982):

1) Vereda El Provenir (Rioblanco, Tolima, Colombia) $\left(03^{\circ} 34^{\prime} 14.8^{\prime \prime} \mathrm{N}, 75^{\circ} 38^{\prime} 59.5^{\prime \prime} \mathrm{O}\right)$, en la subcuenca del río Anamichú, a $1371 \mathrm{~m} \mathrm{s.} \mathrm{n.} \mathrm{m.;} \mathrm{en} \mathrm{la} \mathrm{zona} \mathrm{se} \mathrm{encuentran}$ cultivos de café, con algunos fragmentos de bosque que representan relictos de coberturas continuas que no superan las tres hectáreas, bosques riparios y vegetación en crecimiento secundario en la zona de vida bosque húmedo premontano. En mayo de 2008 se emplearon cuatro redes de niebla estándar $(12 \mathrm{~m} \times 2.5 \mathrm{~m}, 32 \mathrm{~mm}$ de ojo de malla), distribuidas en diferentes hábitats de la zona, las cuales estuvieron abiertas entre las 6:00 y 11:00 horas y 14:00-18:00 horas (Molina-Martínez et al., 2008).

2) Vereda Vega Chiquita (Chaparral, Tolima; $\left.03^{\circ} 48^{\prime} 51.1^{\prime \prime} \mathrm{N}, 75^{\circ} 35^{\prime} 24.8^{\prime \prime} \mathrm{O}\right)$, en el margen del río Amoyá a $1132 \mathrm{~m} \mathrm{s.} \mathrm{n.} \mathrm{m.;} \mathrm{comprende} \mathrm{una} \mathrm{zona} \mathrm{de} \mathrm{reforestación}$ con especies como el cedro rosado (Cedrela odorata) y acacio forrajero (Leucaena sp.) y cultivos abandonados de cacao (Teobroma cacao) en una zona de vida bosque húmedo premontano. En septiembre de 2016 se emplearon cinco redes de niebla estándar $(12 \mathrm{~m} \times 2.5 \mathrm{~m}$, $32 \mathrm{~mm}$ de ojo de malla), distribuidas en diferentes hábitats de la zona, operadas entre 06:00 -11:00 horas y 15:30-17:30 horas.

3) Periferia de la zona urbana de Chaparral a $942 \mathrm{~m} \mathrm{~s}$. n. $\mathrm{m} ., 4 \mathrm{~km}$ al Oeste del casco urbano $\left(03^{\circ} 43^{\prime} 57.5^{\prime \prime} \mathrm{N}\right.$, $75^{\circ} 31^{\prime} 28.5^{\prime \prime} \mathrm{O}$ ); comprende un bosque ripario entre $70 \mathrm{~m}$ y $140 \mathrm{~m}$ de ancho, en una matriz de terreno plano con pasturas; se encuentra en una zona de transición entre bosque seco tropical a bosque húmedo premontano. En junio de 2018 se recorrió un transecto de $1500 \mathrm{~m}$ de longitud entre las 06:00 y 11:00 horas. Los especímenes recolectados están depositados en la Colección Zoológica de la Universidad del Tolima-Ornitología (CZUT-OR). 
Se consultaron los registros disponibles de C. leucorrhoa en los portales de eBird (https:/ / ebird.org/species/ whbman2), Global Biodiversity Information Facility (GBIF) (https:/ / doi.org/10.15468/dl.lamvia), Instituto de Ciencias Naturales de la Universidad Nacional (http:/ / www.biovirtual.unal.edu.co/es/colecciones/ result/species/Corapipo \%20leucorrhoa/birds/) y la Colección Zoológica de la Universidad del TolimaOrnitología (CZUT-OR), información con la que se elaboró una base de datos curada con la georreferencia de cada registro. Para la elaboración del mapa de distribución histórico se tomó en cuenta la información de Mapas Aves de Colombia (https:/ / sites.google.com/site/mapasavescolombia/paserinos-2/pipridae-saltarines).
Con las bases de datos generamos un modelo de distribución potencial de C. leucorrhoa para Colombia, usando el algoritmo MaxEnt (Merow, 2013) y las capas de 19 datos bioclimáticos a $1 \mathrm{~km}^{2}$ de resolución disponibles en la base de datos de WORLDCLIM (http:/ / www.worldclim.org; Hijmans et al., 2005). El MaxEnt determina la relación entre las variables bioclimáticas y los registros de las especies, tratando de encontrar la distribución de probabilidad de máxima entropía (cercana a la uniforme), sujeta a limitaciones impuestas por la información disponible en torno a la distribución observada de la especie y las condiciones ambientales del área de estudio (Guisan \& Thuiller, 2005). El mapa obtenido fue exportado a QGIS 3.6 y se generó el mapa final del

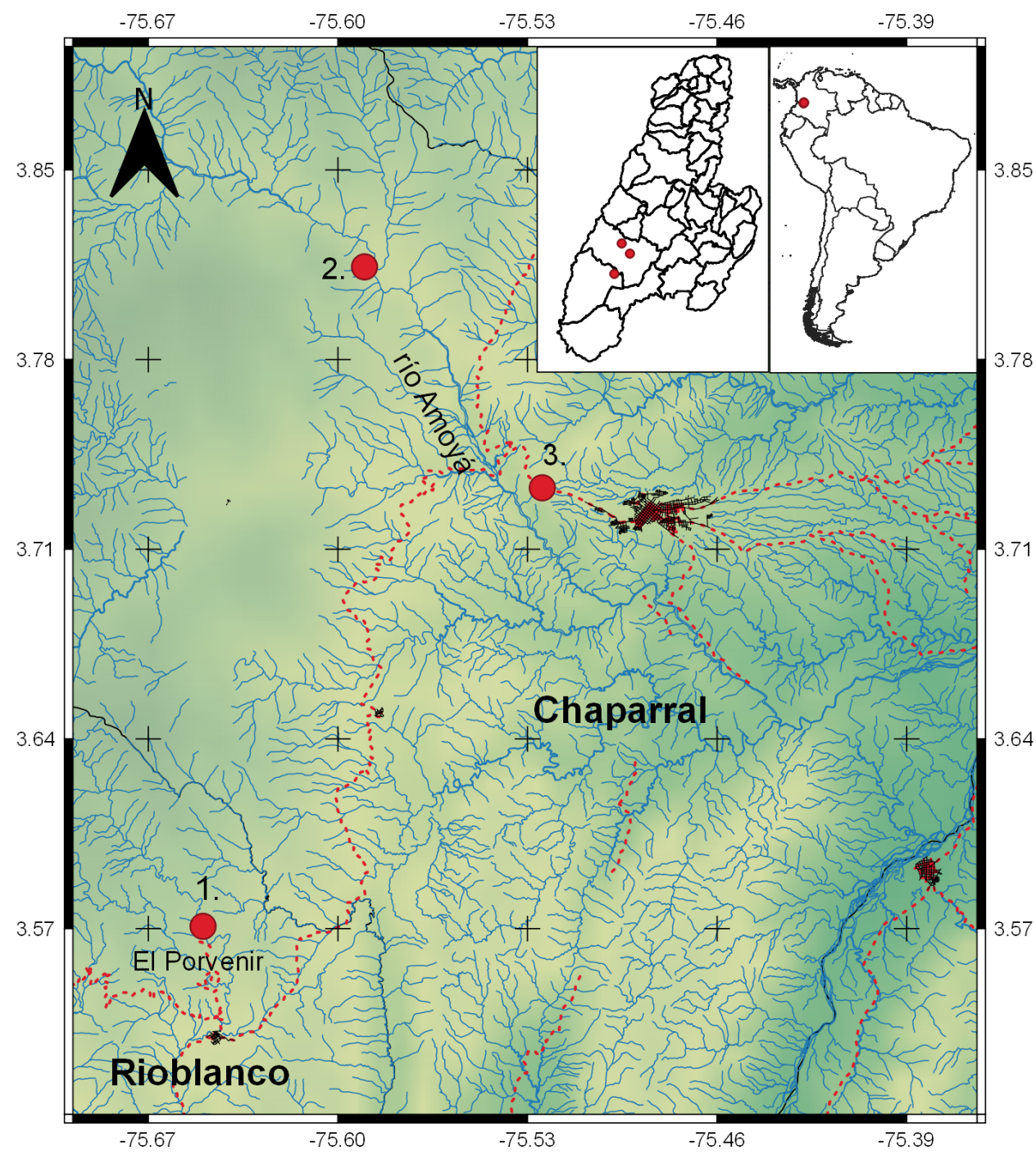

Figura 1. Localidades con registros de Corapipo leucorrhoa en el sur del alto valle del río Magdalena, Colombia. 1, Vereda El Porvenir (Rioblanco, Tolima); 2, Vereda Vega Chiquita (Chaparral, Tolima); 3, periferia del casco urbano de Chaparral. 
modelo potencial de la presencia de C. leucorrhoa. El modelo obtenido con Maxent fue evaluado estadísticamente analizando el área bajo la curva (AUC: Area Under the Curve). El AUC varía de 0 a 1; cuanto más cercano a 1 sea el valor de AUC quiere decir que el modelo tiene buen desempeño, mientras que valores cercanos o por debajo de 0.5 indican que el modelo no es más informativo que lo obtenido por azar.

\section{Resultados}

Los registros de C. leucorrhoa hacia el sur del valle alto del Magdalena fueron los siguientes: 1) un individuo macho adulto capturado el 5 de mayo del 2008 en la vereda el Porvenir (Rioblanco, Tolima; (CZUT-OR 0930; Molina-Martínez et al., 2008; Molina-Martínez et al., 2015); 2) una hembra adulta capturada el 10 de septiembre del 2016 en la vereda de Vega Chiquita (Chaparral, Tolima; CZUT-OR 1454; Losada-Prado et al., 2016)
(Figura 2); 3) una hembra que se observó forrajeando entre el follaje en un borde de bosque en la periferia del casco urbano de Chaparral, el 7 de junio del 2018 (Losada-Prado et al., 2018).

El modelo de distribución para Corapipo leucorrhoa obtuvo un AUC de 0.942 , lo que significa que el modelo tiene un buen ajuste. Las predicciones realizadas para esta especie indicaron que podría tener una distribución continua con una mayor presencia en la región del Magdalena medio, disminuyendo gradualmente hacia los piedemontes del valle alto del Magdalena (Tolima, Cundinamarca), por lo cual esta región debe ser incluida como parte de su distribución geográfica para Colombia, pues el modelo muestra que la probabilidad de presencia de la especie en la región es mayor al $70 \%$ (Figura 2). En general las variables con mayor contribución al modelo de distribución de C. leucorrhoa son las que se encuentran relacionadas con la temperatura y la estacionalidad de la precipitación, con porcentajes de $20.9-7.0 \%$ (Tabla 1 ).
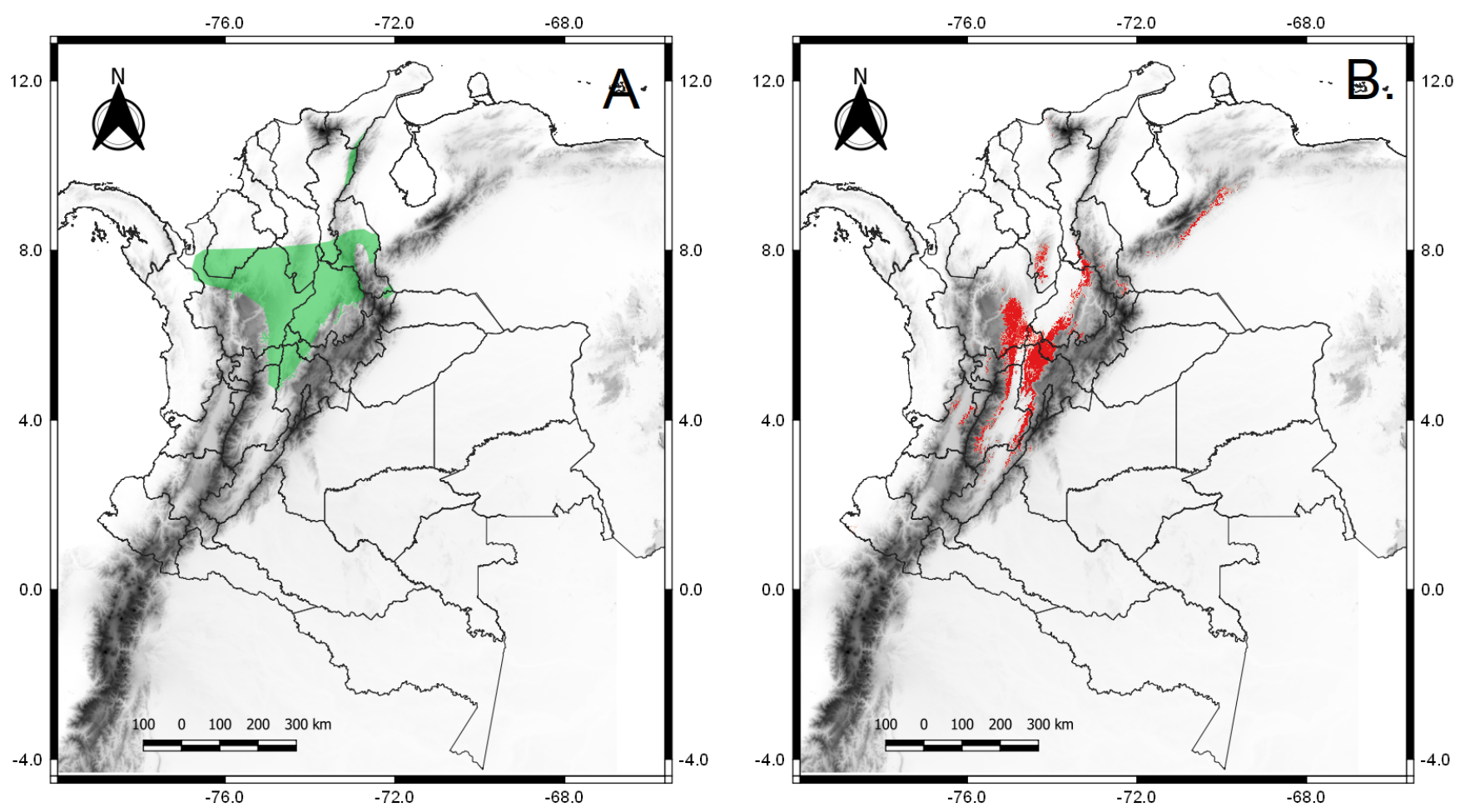

Figura 2. Distribución de Corapipo leucorrhoa. A, distribución histórica (https:/ / sites.google.com/site/mapasavescolombia/paserinos-2/pipridae-saltarines); B, distribución modelada en MaxEnt. 
Tabla 1. Porcentaje de contribución de las variables bioclimáticas usadas en la modelación de la distribución de Corapipo leucorrhoa.

\begin{tabular}{ll}
\hline Variable & $\begin{array}{c}\text { Porcentaje de } \\
\text { contribución }\end{array}$ \\
\hline BIO3 = Isotermalidad (BIO2 $/$ BIO7) ${ }^{*}$ 100) & 20.9 \\
\hline BIO5 = Temperatura máxima del mes más cálido & 18.1 \\
\hline BIO10 = Temperatura media del trimestre más cálido & 13.4 \\
\hline BIO15 = Estacionalidad de precipitación (Coeficiente de variación) & 12.4 \\
\hline BIO2 = Rango diurno medio (Promedio de mensual (temperatura máxima - temperatura mínima)) & 8.5 \\
\hline BIO8 = Temperatura media del trimestre más húmedo & 7.0 \\
\hline BIO4 = Estacionalidad de temperatura (desviación estándar * 100) & 3.8 \\
\hline BIO16 = Precipitación del trimestre más húmedo & 3.6 \\
\hline BIO18 = Precipitación del trimestre más cálido & 3.4 \\
\hline BIO7 = Rango anual de temperatura (BIO5-BIO6) & 2.7 \\
\hline BIO17 = Precipitación del trimestre más seco & 1.8 \\
\hline BIO11 = Temperatura media del trimestre más frío & 1.3 \\
\hline BIO19 = Precipitación del trimestre más frío & 1.3 \\
\hline BIO12 = Precipitación anual & 0.7 \\
\hline BIO6 = Temperatura mínima del mes más frío & 0.6 \\
\hline BIO13 = Precipitación del mes más húmedo & 0.3 \\
\hline BIO1 = Temperatura media anual & 0.2 \\
\hline BIO9 = Temperatura media del trimestre más seco & 0.0 \\
\hline BIO14 = Precipitación del mes más seco & 0.0 \\
\hline
\end{tabular}

\section{Discusión}

Los nuevos registros de esta especie al sur del valle alto del Magdalena podrían corresponder a dos escenarios: 1) Roselli et al. (1994) y Boyle et al. (2010) han demostrado migraciones $\mathrm{y} / \mathrm{o}$ dispersiones altitudinales en especies similares, como en $C$. altera en bosques húmedos. Sin embargo, en el caso de C. leucorrhoa no hay evidencias de algún tipo de dispersión altitudinal o latitudinal, debido a la carencia de estudios ecológicos de esta especie. 2) Los reportes de C. leucorrhoa que están al norte y al sur del valle alto del Magdalena no serían registros aislados, sino que podrían indicar que su distribución aún no es bien conocida o que podría presentar una extensión de su distribución, ya que los nuevos registros se encuentran a $110 \mathrm{~km}$ lineales de la distribución presentada hasta ahora para la especie (Molina-Martínez et al., 2015).
El modelamiento en MaxEnt muestra que C. leucorrhoa y las variables bioclimáticas muestran que hay una mayor probabilidad de presencia en los piedemontes del alto valle del Magdalena. Esto puede deberse a que esta especie haya encontrado un nicho ecológico que haya favorecido su dispersión y su adaptación a las condiciones climáticas en la zona. Esta especie podría presentar preferencias por transiciones de bosques secos a bosques húmedos premontanos dentro de los rangos altitudinales reportados (2001500 m s. n. m.; Hilty \& Brown, 2001; Restall et al., 2006; Ayerbe-Quiñones, 2018). Además, en los cañones del río Amoyá (Chaparral, Tolima) y la cuenca del río Anamichú (Rioblanco, Tolima) podrían ser zonas propicias para el establecimiento de esta especie, por la presencia de algunos bosques relictuales y matorrales cercanos a estos ríos, que podrían generar microclimas más húmedos. La zona oriental del departamento del 
Tolima debería ser mejor muestreada, ya que está dentro de la distribución potencial modelada, con una alta probabilidad de presencia de esta especie.

\section{Agradecimientos}

Agradecemos a CORTOLIMA, al Grupo de Investigación en Zoología, al Comité Central de Investigaciones de la Universidad del Tolima e ISAGEN con el convenio No. 47/0821 por el apoyo en la ejecución en el proyecto "Evaluación limnológica del río Amoyá y sus tributarios La Arenosa, La Alcancía y La Virginia; el monitoreo de fauna vertebrada silvestre asociadas a la Central Hidroeléctrica río Amoyá La Esperanza y el seguimiento a la colisión de aves en la línea de transmisión Amoyá-Tuluní" en sus diferentes fases, durante las cuales se obtuvieron los datos de este estudio.

\section{Referencias}

American Ornithologists' Union. (1998). Checklist of North American Birds, 7th edition. Washington D.C.: American Ornithologists' Union. 829 pp.

Ayerbe-Quiñones, F. (2018). Avifauna Colombiana. Bogotá D. C.: Wildlife Conservation Society. 442 pp.

Boyle, W. A., Norris, D. R. \& Guglielmo, C. G. (2010). Storms drive altitudinal migration in a tropical bird. Proceedings of the Royal Society, B 277(1693), 25112519. https://doi.org/10.1098/rspb.2010.0344

Chaparro-Herrera, S., Echeverry-Galvis, M. A., Córdoba-Córdoba, S. \& Sua-Becerra, A. (2013). Listado actualizado de las aves endémicas y casi-endémicas de Colombia. Biota Colombiana, 14(2), 235-272. http:// doi.org/10.15472/tozuue

Guisan A. \& Thuiller W. (2005). Predicting species distribution: offering more than simple habitat models. Ecology Letters, 8, 993-1009. https:/ / doi.org/10.1111/j.1461-0248.2005.00792.x

Hijmans, R. J., Cameron, S. E., Parra, J. L., Jones, P. \& Jarvis, A. (2005). Very high resolution interpolated climate surfaces for global land areas. International Journal of Climatology, 25, 1965-1978. https://doi.org/10.1002/joc.1276

Hilty, S. L. \& W. Brown, L. (2001). Guía de las aves de Colombia. Edición en español. Cali, Colombia: American Bird Conservation (ABC). $1030 \mathrm{pp}$.

Holdridge, L. (1982). Ecología Basada en Zonas de Vida. San José, Costa Rica: Tropical Science Center. 216 pp.
Losada-Prado, S., Gaitán-García, C. D. \& Vargas, H. (2015). Aves. En Reinoso-Flórez, G., Villa-Navarro, F. A. \& Losada-Prado, S. (Eds.). Evaluación limnológica, monitoreo de fauna vertebrada silvestre y colisión de aves en la línea de transmisión Amoyá-Tuluní, Central Hidroeléctrica Rio Amoyá, La Esperanza. Pp: 130-144. Ibagué, Colombia: ISAGEN y Grupo de Investigación en Zoología, Universidad del Tolima.

Losada-Prado, S., Gaitán-García, C. D. \& Vargas, H. (2016). Aves. En Reinoso-Flórez, G., Villa-Navarro, F. A., Losada-Prado, S. \& Guevara-Cardona, G. (Eds.). Evaluación limnológica del río Amoyá y sus tributarios La Arenosa, La Alcancía y La Virginia; el monitoreo de fauna vertebrada silvestre asociadas a la Central Hidroeléctrica río Amoyá La Esperanza y el seguimiento a la colisión de aves en la línea de transmisión Amoyá-Tuluní. Pp: 193-217. Ibagué, Colombia: ISAGEN y Grupo de Investigación en Zoología, Universidad del Tolima.

Losada-Prado, S., Gaitán-García, C. D. \& Villabón, C. (2018). Seguimiento a la colisión de aves en la línea de transmisión Amoyá-Tuluní. En Reinoso-Flórez, G., Villa-Navarro, F. A., Losada-Prado, S. \& GuevaraCardona, G. (Eds.). Evaluación limnológica del río Amoyá y sus tributarios La Arenosa, La Alcancía y La Virginia; el monitoreo de fauna vertebrada silvestre asociadas a la Central Hidroeléctrica río Amoyá La Esperanza y el seguimiento a la colisión de aves en la línea de transmisión Amoyá-Tuluní. Pp: 3-22. Ibagué, Colombia: ISAGEN y Grupo de Investigación en Zoología, Universidad del Tolima.

Losada-Prado, S., Moreno-Palacios, M. C \& PachecoVargas, G. F. (2014). Aves. En Reinoso-Flórez, G., Villa-Navarro \& Losada-Prado, S. (Eds.). Monitoreo de vertebrados terrestres (anfibios, reptiles, aves $y$ mamíferos) asociados a la Central Hidroeléctrica río Amoyá La Esperanza. Pp 31-46. Ibagué, Colombia: ISAGEN y Grupo de Investigación en Zoología, Universidad del Tolima.

Merow, C., Smith, M. J., \& Silander, J. A. (2013). A practical guide to MaxEnt for modeling species' distributions: what it does, and why inputs and settings matter. Ecography, 36, 1058-1069. https:// doi.org/10.1111/j.1600-0587.2013.07872.x

Molina-Martínez, Y. G., Díaz, H. M. \& Gómez, C. A. Aves. (2008). En Reinoso-Flórez, G., Villa-Navarro, F. A., Esquivel, H. E., García-Melo, J. E. \& VejaranoDelgado, M. A. (Eds.). Biodiversidad Faunística y Florística de la cuenca mayor del rio Saldaña (subcuenca Anamichú). Biodiversidad Regional Fase IV. Pp: 333470. Ibagué, Colombia: ISAGEN y Grupo de Investigación en Zoología, Universidad del Tolima. 
Molina-Martínez, Y. G., García-Melo, J. E. \& LosadaPrado, S. (2015). Evaluación rápida de las aves de la parte baja de la cuenca del río Anamichú, municipio de Rioblanco-Tolima. Tumbaga, 10(2), 72-93.

Remsen, J. V., Areta, J. I., Cadena, C. D., Claramunt, S., Jaramillo, A., Pacheco, J. F., Robbins, M. B., Stiles, F. G., Stotz, D. F. \& Zimmer, J. (2019). A classification of the bird species of South America. American Ornithologists' Union. http://www.museum.lsu.edu/ Remsen/

Restall, R., Rodner, C. \& Lentino, M. (2006). Birds of Northern South America: an identification guide, 2. Plates and maps. Londres: Yale University Press. 656 pp.
Ridgely, R. S. \& Tudor, G. (1994). The birds of South America, Vol. 2. Austin, USA: University Texas Press. $950 \mathrm{pp}$.

Rosselli, L., Vásquez, P. \& Ayub, I. (2002). The courtship displays and social system of the White-ruffed Manakin in Costa Rica. Wilson Bulletin, 114(2), 165-178. http://www.jstor.org/stable/4164438

Sibley, C. G. \& Monroe, B. L. (1990). Distribution and Taxonomy of Birds of the World. New Haven, USA: Yale University Press. 1136 pp. 
Cristhian David Gaitán García

Universidad del Tolima

Ibagué, Colombia

cdgaitang@ut.edu.co

https://orcid.org/0000-0002-3852-8615

\section{Sergio Losada Prado}

Universidad del Tolima

Ibagué, Colombia

slosada@ut.edu.co
Modelamiento de la distribución potencial de Corapipo leucorrhoa (Pipridae) en el valle alto del Magdalena, Tolima, Colombia

Citación del artículo: Gaitán-García, C.D. \& Losada-Prado, S. (2020). Modelamiento de la distribución potencial de Corapipo leucorrhoa (Pipridae) en el valle alto del Magdalena, Tolima, Colombia. Biota Colombiana, 21(1), 109-116. DOI: 10.21068/c2020.v21n01a08.

Recibido: 24 de mayo 2019

Aprobado: 12 de noviembre 2019 